American Journal of Applied Sciences 5 (10): 1322-1328, 2008

ISSN 1546-9239

(C) 2008 Science Publications

\title{
Input-Output Feedback Linearization Cascade Controller Using Genetic Algorithm for Rotary Inverted Pendulum System
}

\author{
Iraj Hassanzadeh, Saleh Mobayen and Abbas Harifi \\ Department of Control Engineering, Research Laboratory of Robotics, \\ Faculty of Electrical and Computer Engineering, University of Tabriz, Tabriz, Iran
}

\begin{abstract}
The Rotary Inverted Pendulum (RIP) system is a significant classical problem of control engineering which has been investigated in the past decades. This study presents an optimum InputOutput Feedback Linearization (IOFL) cascade controller utilized Genetic Algorithm (GA). Due to the non-minimum phase behavior of the system, IOFL controller leads to unstable internal dynamics. Therefore a cascade structure is proposed consisting IOFL controller for inner loop with PD controller forming the outer loop. The primary design goal is to balance the pendulum in an inverted position. The control criterion is to minimize the Integral Absolute Error (IAE) of system angles. By minimizing the objective function related to IAE using Binary Genetic Algorithm (BGA), the optimal controller parameters can be assigned. The results verified capability and competent characteristics of the proposed controller. The method can be considered as a promising way for control of various similar nonlinear and under-actuated systems.
\end{abstract}

Key words: Rotary inverted pendulum, input-output feedback linearization, binary genetic algorithm, under-actuated system, nonlinear model

\section{INTRODUCTION}

Feedback linearization is a control design approach for nonlinear systems which attracted lots of research in recent years ${ }^{[1-3]}$. The central idea is to algebraically transform nonlinear systems dynamics into (fully or partially) linear ones, so that linear control techniques can be applied.

Even though the design of controllers for nonlinear systems has been well researched, the conventional input-output linearization techniques will perform very poorly when it deals with output tracking ${ }^{[2,4]}$. The inputoutput feedback linearization law consists of inverting the system dynamics given a nonlinear change of coordinates and a feedback law. IOFL is a systematic way to linearize globally dynamics of systems ${ }^{[4]}$.

In this research, the IOFL technique is proposed as a systematic method for designing a pre-stabilizer. Then, another PD controller, optimized using GA is applied to the pre-stabilized system to prevent unstable internal dynamics.

Unfortunately, it has been difficult to tune the primary controller gains of this controller accurately. The ability of using numerical methods for efficiently and accurately characterizing the quality of a particular design has excited control engineers to apply stochastic global optimizers.
Over the past years, several heuristic methods are employed for tuning of controllers. In this study, we present the Binary Genetic Algorithm for designing controller parameters for the new input and formulate the design procedure as an optimization problem ${ }^{[5-7]}$.

\section{ROTARY INVERTED PENDULUM SYSTEM}

The rotary inverted pendulum system is a wellknown test platform for evaluating various control algorithms. It has also some significant real life applications such as pointing control, aerospace vehicles control, robotics and so on ${ }^{[8-11]}$.

The system consists of a rotary arm and a pendulum where the rotary arm is actuated by a motor with the objective of balancing the pendulum in the inverted position. A schematic diagram of the RIP system is represented in Fig. 1, where $u, l_{p}, m_{p}, \alpha, r, \theta$ and $\mathrm{J}_{\mathrm{b}}$ are the motor input, the pendulum length, the pendulum mass, the pendulum angle, the arm length, the arm angle and effective mass moment of inertia, respectively.

The plane of the pendulum is orthogonal to the radial arm. Figure 2 shows the RIP system built in robotics research lab in our department. Also, the block diagram of whole system is shown in Fig. 3. The system consists of a geared DC motor (Mitsubishi, $18 \mathrm{~V}$

Corresponding Author: Iraj Hassanzadeh, Faculty of Electrical and Computer Engineering, University of Tabriz, Tabriz, Iran 


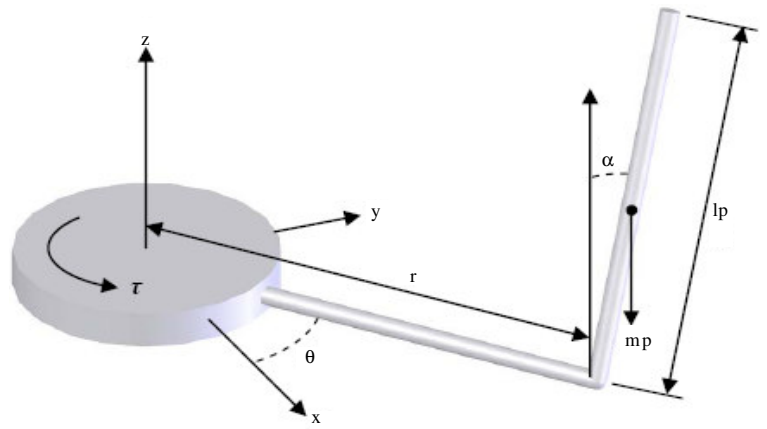

Fig. 1: Schematic view of the RIP system

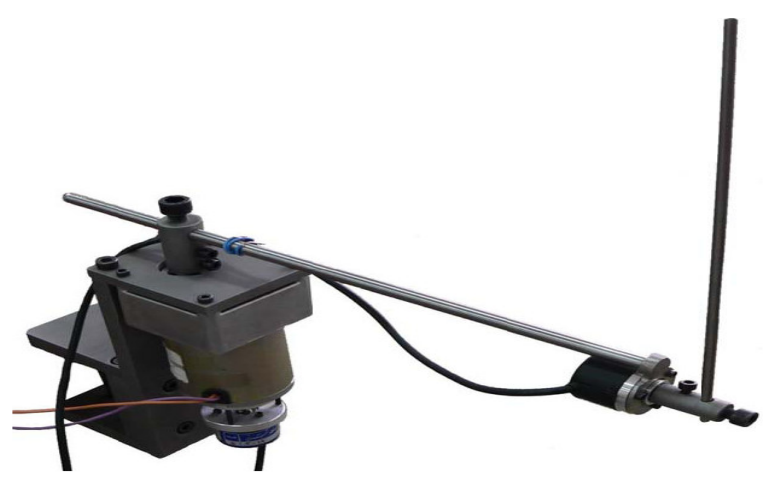

Fig. 2: Built in RIP system (advanced robotics research lab)

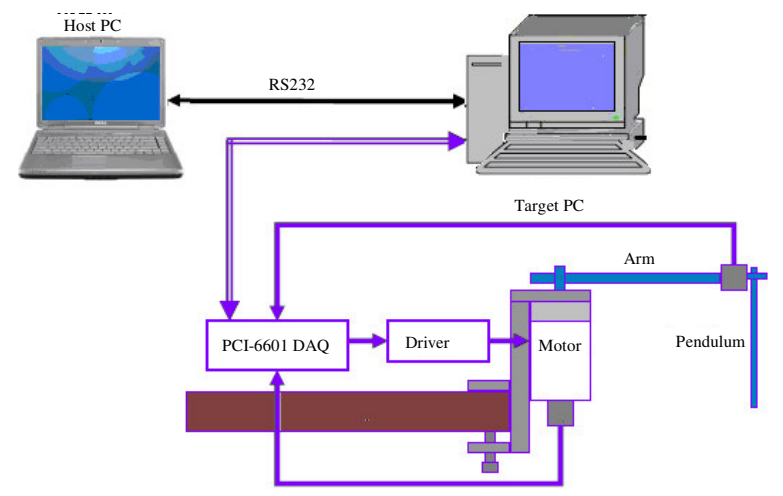

with gear ratio of 13:1) as an actuator and two encoders (E40s-Autonics, with 1024 PPR resolution) to measure the arm and pendulum angles and the master side is comprised of a PC P4-1.7 GHz.

The $\mathrm{xPC} \operatorname{target}^{\circledR}$ toolbox from MATLAB ${ }^{\circledR}$ provides the hardware in the loop property, so this package is applied to control the system. The slave side consists of the RIP system, an integrated high powerrated PWM driver (to drive the DC motor), a PCI-6602 encoder reader and a slave computer PC P3-750 MHz.

The PCI-6602 encoder reader mounted in the slave side computer reads the pendulum and rotary arm angles. Also, two bits of digital output channels of PCI6602 are used to command the PWM driver.

Here, nonlinear dynamic equations of the RIP system considering backlash and friction effects are presented. The RIP dynamics are governed by ${ }^{[10,11]}$ :

$$
\begin{gathered}
\left(\mathrm{A}+\mathrm{B} \sin ^{2} \alpha\right) \ddot{\theta}+(\mathrm{C} \cos \alpha) \ddot{\alpha}-(\mathrm{C} \sin \alpha)(\dot{\alpha})^{2}+ \\
(2 \mathrm{~B} \sin \alpha \cos \alpha) \dot{\alpha} \dot{\theta}+\mathrm{F} \dot{\theta}+\mathrm{G} \cdot \operatorname{sign}(\dot{\theta})+\mathrm{H} \cdot \theta=\mathrm{I} . \mathrm{u}
\end{gathered}
$$

$$
\ddot{\mathrm{B}} \ddot{\alpha}+(\mathrm{C} \cos \alpha) \ddot{\theta}-(\mathrm{B} \sin \alpha \cos \alpha)(\dot{\theta})^{2}-\mathrm{D} \sin \alpha+\mathrm{E} \dot{\alpha}=0
$$

where,

$$
\begin{aligned}
& \mathrm{A}=\mathrm{m}_{\mathrm{p}} \mathrm{r}^{2}+\mathrm{J}_{\mathrm{b}} \\
& \mathrm{B}=\frac{1}{3} \mathrm{~m}_{\mathrm{p}} \mathrm{l}_{\mathrm{p}}^{2} \\
& \mathrm{C}=\frac{1}{2} \mathrm{~m}_{\mathrm{p}} \mathrm{rl}_{\mathrm{p}} \\
& \mathrm{D}=\frac{1}{2} \mathrm{~m}_{\mathrm{p}} \mathrm{g} \mathrm{l}_{\mathrm{p}}
\end{aligned}
$$

By defining $\mathrm{x}_{1}=\alpha, \mathrm{x}_{2}=\dot{\alpha}, \mathrm{x}_{3}=\theta$ and $\mathrm{x}_{4}=\dot{\theta}$, the nonlinear model of Eq. 1 and 2 can be represented as,

$$
\dot{\mathrm{x}}=\mathrm{F}(\mathrm{x})+\mathrm{G}(\mathrm{x}) \cdot \mathrm{u}
$$

with

Fig. 3: Block diagram of whole system

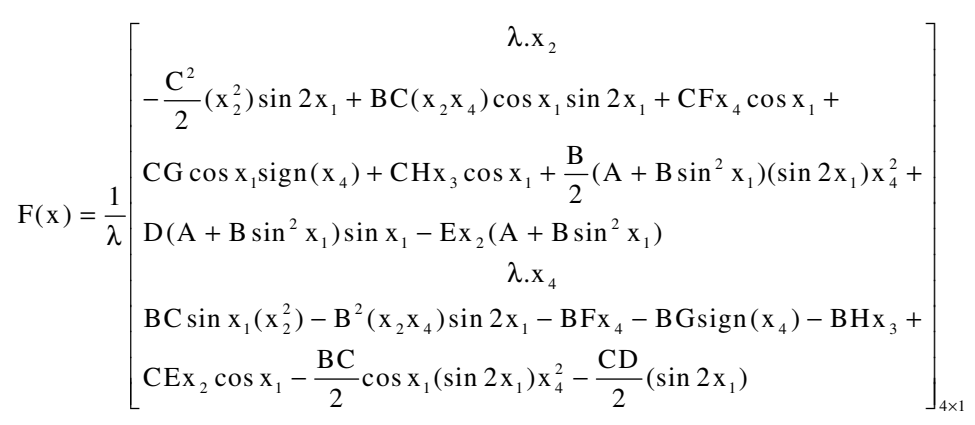


Am. J. Applied Sci., 5 (10): 1322-1328, 2008

Table 1: Parameters of the RIP system

\begin{tabular}{ll}
\hline Parameters & Values \\
\hline $\mathrm{A}$ & 3.29 \\
$\mathrm{~B}$ & 0.1252 \\
$\mathrm{C}$ & 0.2369 \\
$\mathrm{D}$ & 6.052 \\
$\mathrm{E}$ & 0.0132 \\
\hline & $\mathrm{G}(\mathrm{x})=\frac{1}{\lambda}\left[\begin{array}{c}0 \\
-\mathrm{CI} \cos \mathrm{x}_{1} \\
0 \\
\mathrm{~B} . \mathrm{I}\end{array}\right]$
\end{tabular}

Where,

$$
\lambda=\mathrm{BA}+\left(\mathrm{B} \sin \mathrm{x}_{1}\right)^{2}-\left(\mathrm{C} \cos \mathrm{x}_{1}\right)^{2}
$$

Then, the above model is severely nonlinear since the matrices $\mathrm{F}$ and $\mathrm{G}$ are state dependent.

The parameters of nonlinear model of the system are identified using GA method and represented in Table 1.

\section{INPUT-OUTPUT FEEDBACK LINEARIZATION METHOD IN BRIEF}

The input-output feedback linearization of singleinput nonlinear systems is described by the state space representation as follows ${ }^{[1]}$ :

$$
\begin{aligned}
& \dot{x}=f(x)+g(x) u \\
& y=h(x)
\end{aligned}
$$

The control objective is to make the output y track a desired trajectory $y_{d}$ while keeping the states bounded. An obvious complexity of this model is that the output $\mathrm{y}$ is indirectly related to the input $\mathrm{u}$ through the state variable $\mathrm{x}$ and the nonlinear state equations. The diagram of this method is shown in Fig. 4. The difficulty of the tracking control design can be decreased by finding a simple and direct relation between the system output $y$ and the control input $u$.

Therefore, this idea constitutes an intuitive basis for the input-output linearization approach to nonlinear control design. In this method, a linear differential relation is generated between the output, y and a new input, v. By differentiating the output equation, we have

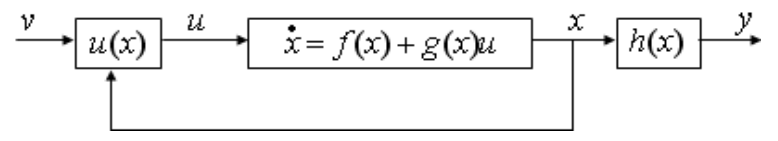

Fig. 4: Input-output feedback linearization $\dot{y}=\frac{\partial h}{\partial x} \dot{x}=\frac{\partial h}{\partial x}(f(x)+g(x) u)=L_{f} h(x)+L_{g} h(x) u$

Then, the control law can be defined as

$$
\mathrm{u}=\frac{1}{\mathrm{~L}_{\mathrm{g}} \mathrm{h}(\mathrm{x})}\left(\mathrm{v}-\mathrm{L}_{\mathrm{f}} \mathrm{h}(\mathrm{x})\right)
$$

that renders the linear differential equation

$$
\dot{y}=v
$$

The proposed controller is based on the theory of input-output feedback linearization. Pendulum angle $(\alpha)$ is considered as the output (y). To declare y or its derivatives in terms of $\mathrm{u}$, some manipulation and evaluation of Eq. 4 are required.

$$
\ddot{y}=F_{2}(x)+G_{2}(x) \cdot u
$$

The input-output feedback linearization law can be formulated as:

$$
\mathrm{u}=\frac{\mathrm{v}-\mathrm{F}_{2}(\mathrm{x})}{\mathrm{G}_{2}(\mathrm{x})}
$$

where, $\mathrm{v}$ is the new input. Then the nonlinearity in Eq. 12 is canceled and a simple linear double-integrator relationship between the output and the new input $\mathrm{v}$ is obtained as follows:

$$
\ddot{y}=v
$$

Now, the whole system is linear and consequently the design of a tracking controller is quite easy. The asymptotically stability of the RIP system is guaranteed using definition of the new input $\mathrm{v}$ and $\mathrm{y}_{\mathrm{ref}}$ as;

$$
\begin{gathered}
\mathrm{v}=\mathrm{K}_{\mathrm{pi}}\left(\mathrm{y}_{\text {ref }}-\mathrm{y}\right)+\mathrm{K}_{\mathrm{di}}\left(\dot{\mathrm{y}}_{\mathrm{ref}}-\dot{\mathrm{y}}\right) \\
\mathrm{y}_{\mathrm{ref}}=\mathrm{K}_{\mathrm{po}} \theta+\mathrm{K}_{\mathrm{do}} \dot{\theta}
\end{gathered}
$$

To obtain optimal results, we employ GA method for adjusting controller parameters based on an introduced cost function which discuss for further. 


\section{BINARY GENETIC ALGORITHM OVERVIEW}

GA is an optimization and search method based on the principles of natural genetics and natural selection and is widely recognized as an effective search paradigm in many areas ${ }^{[12,15]}$. The algorithm first was described by John Holland (1975) over the course of the 1960s and 1970s and popularized by David Goldberg, who was able to solve a difficult problem such as the controlling of gas-pipeline transmission for his thesis ${ }^{[6]}$. The biological basis of this algorithm is Darwinian natural selection that is elimination of weak and inefficient elements by optimal and near-optimal individuals and maintaining and recombination of features of good individuals to make new generations and better individuals. BGA introduces variables as an encoded binary string and works with the binary strings to arrive at the global best solution and maximize the fitness, i.e., minimize the cost function ${ }^{[13-15]}$.

The optimization process is performed in cycles called generations and during each generation, a set of new chromosomes is created using the crossover, inversion and mutation processes and only the best individuals (chromosomes) are allowed to survive to the next cycle of reproduction ${ }^{[5]}$.

We set binary genetic algorithm parameters for verifying the performance of the controller in searching the controllers' parameters according to the trial and error manner as follows:

- Population size $=20$

- Crossover rate $=0.5$

- Mutation rate $=0.02$

- Maximum generation $=30$

\section{PROBLEM FORMULATION AND SIMO CONTROLLER DESIGN}

A performance index including Integrated Absolute Error (IAE) of pendulum and arm angles is employed in the paper. The proposed control criterion is as follows:

$$
I A E=I_{\text {pendulum }}+\operatorname{IAE}_{\text {arm }}=\int_{0}^{t_{f}}\left|e_{p}(t)\right| d t+\int_{0}^{t_{f}}\left|e_{a}(t)\right| d t
$$

where, $t_{f}=20$ seconds. BGA approach for searching optimal PD controller parameters is described below:

At first, specify the lower and upper bounds of controller parameters and generate initial, random population of chromosomes. Each chromosome (controller parameters) is sent to Simulink ${ }^{\circledR}$ model.
Then, the value of performance criterion is calculated iteratively in Matlab $^{\circledR}$ environment. After that, cost function is evaluated for each chromosome according to this performance criterion. Comparing the fitness values for all chromosomes, the fittest members of the population are selected. According to the probabilistic method, reproduction is executed and then crossover operation on the reproduced chromosomes is implemented. Then, the algorithm executes mutation operation. At the end of each iteration, program checks the predefined convergence criterion. If the number of iterations reaches the predefined maximum value, program records the latest global best solution and stops.

\section{SIMULATION RESULTS}

The lower and upper bounds of the controller parameters are given in Table 2. The Simulink ${ }^{\circledR}$ block diagram of RIP system with nonlinear controller is shown in Fig. 5. The noise power is 0.001. The simulation results illustrate the effectiveness of the proposed design methodology and the developed theory.

The best controller parameters and system characteristics obtained by BGA are as follows:

Arm controller: $\quad \mathrm{k}_{\mathrm{P}_{\mathrm{o}}}=0.064, \mathrm{k}_{\mathrm{do}}=0.232$

Pendulum controller: $\mathrm{k}_{\mathrm{Pi}}=0.948, \mathrm{k}_{\mathrm{di}}=0.243$

The IAE values for pendulum and arm angles are 17.155 and 12.175 , respectively. Also, IAE for their velocities are 4.1453 and 19.4292 .

Table 2: Range of three controller parameters

\begin{tabular}{lll}
\hline Controller parameters & Lower bounds & Upper bounds \\
\hline $\mathrm{K}_{\mathrm{pi}}$ & 0 & 1 \\
$\mathrm{~K}_{\mathrm{di}}$ & 0 & 1 \\
$\mathrm{~K}_{\mathrm{po}}$ & 0 & 1 \\
$\mathrm{~K}_{\mathrm{do}}$ & 0 & 1 \\
\hline
\end{tabular}

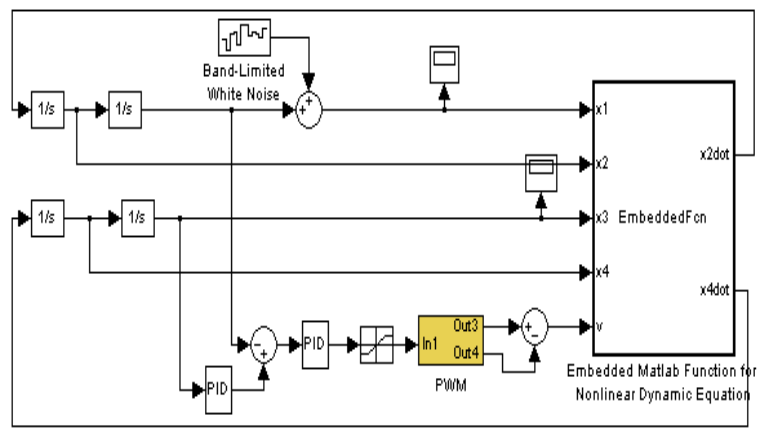

Fig. 5: The Simulink model of RIP system with GAbased IOFL controller 
The signals $y$ and $y_{\text {ref }}$ are shown in Fig. 6. Also, the simulation responses of the pendulum and the arm angles, velocities and accelerations are shown in Fig. 7-12. Simulation results reveal that the proposed method has desirable performance in terms of the integral absolute error.
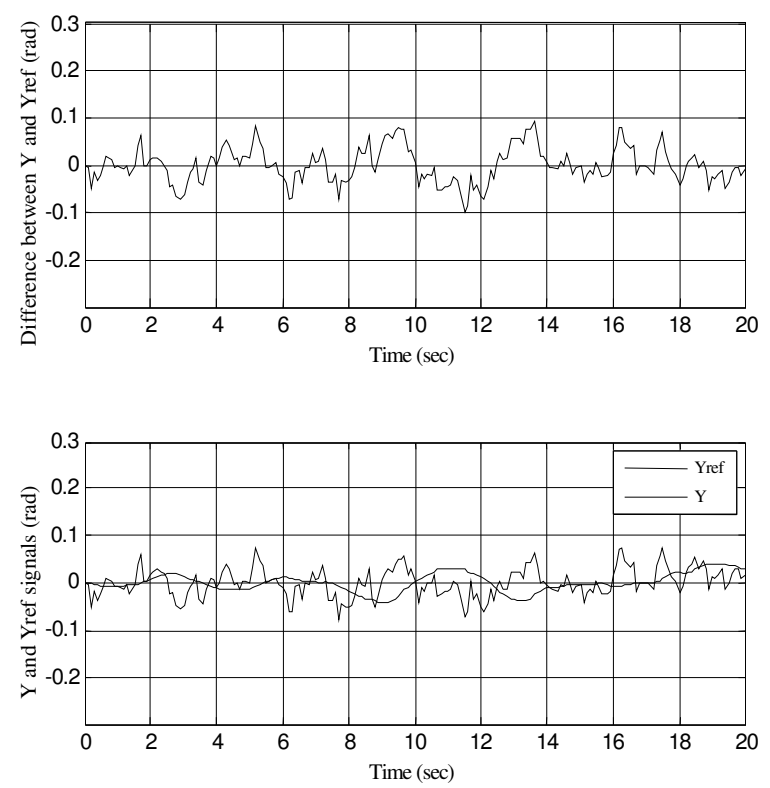

Fig. 6: The signals Y and Yref
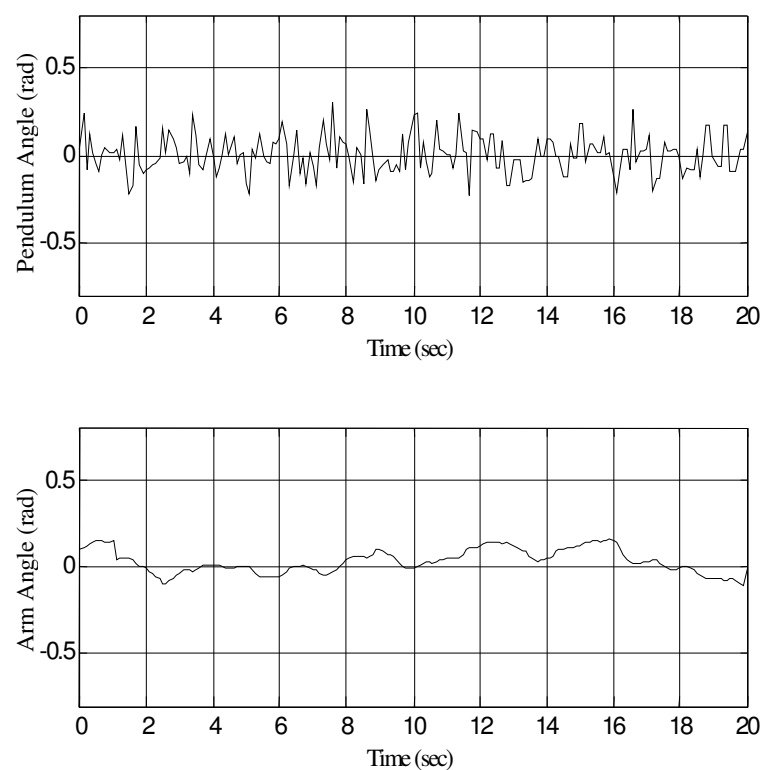

Fig. 7: The arm angle using BGA based IOFL controller
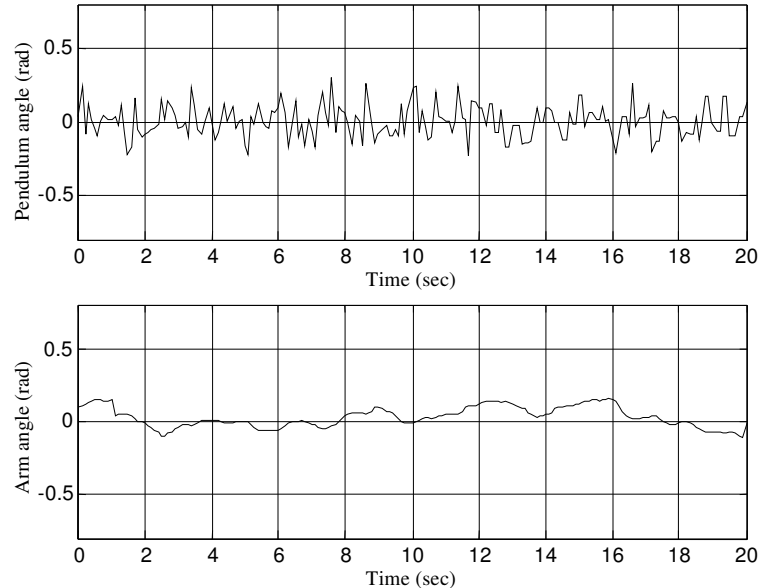

Fig. 8: The pendulum angle using BGA based IOFL controller
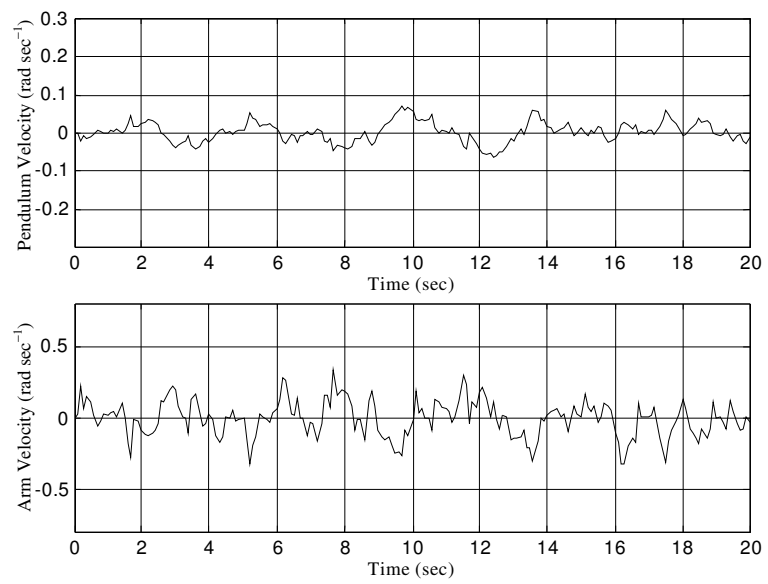

Fig. 9: The arm velocity via BGA based IOFL controller
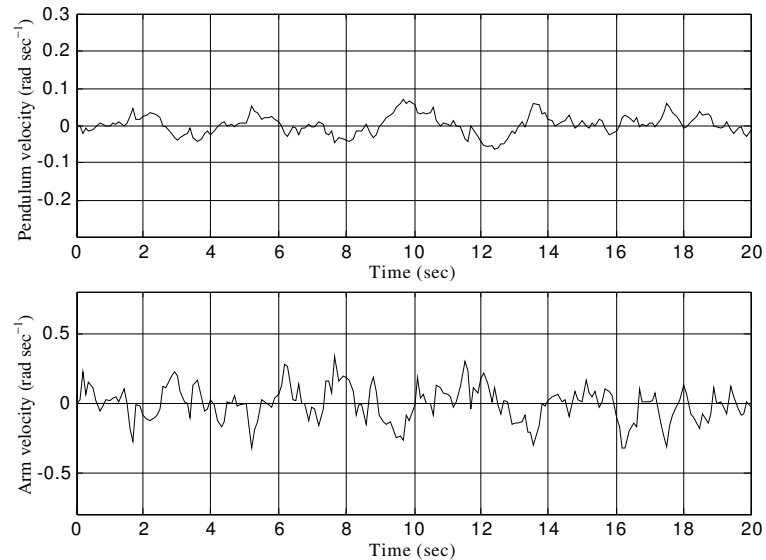

Fig. 10: The pendulum velocity via BGA based IOFL controller 

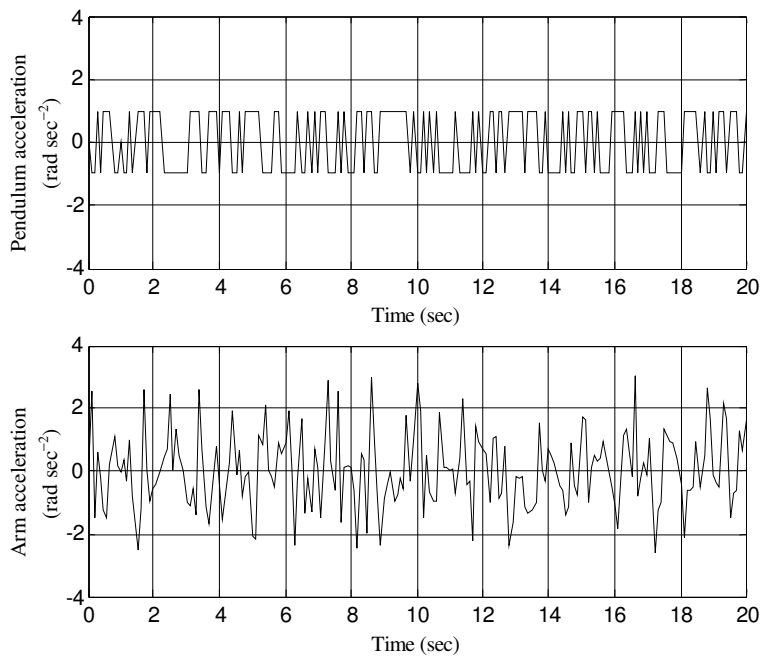

Fig. 11: The arm acceleration via BGA based IOFL controller
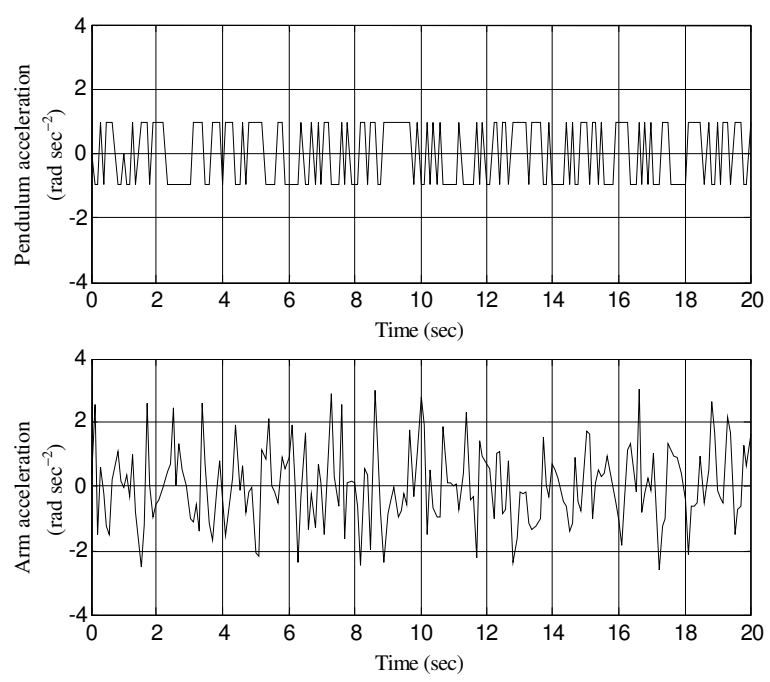

Fig. 12: The pendulum acceleration via BGA based IOFL controller

\section{CONCLUSION}

This study presents an optimum IOFL cascade controller utilized GA. In the cascade scheme, the reference signal $\left(\mathrm{y}_{\text {ref }}\right)$ is adjusted every second to bring the arm back to the origin. With regard to results, the proposed algorithm performs an efficient search for proper controller parameters. This study demonstrates GA method can solve searching and tuning the controller parameters efficiently. The proposed method could be considered as a promising way for nonlinear and under-actuated control systems in general. The topic of our future researches is to utilize other cognitive methods in order to achieve better results for designing controller and improving the performance in real time. Furthermore, tele-operation control of RIP system using haptic device would be our future challenging task.

\section{ACKNOWLEDGEMENT}

This research was financially supported by the Research Affairs at the University of Tabriz.

\section{REFERENCES}

1. Allgöwer, F., 1997. Approximate input-output linearization of non-minimum phase nonlinear systems. European Control Conference, Brussels, Belgium, pp:604.

2. Abdellah Mokhtari, Abdelaziz Benallegue and Boubaker Daachi, 2006. Robust feedback linearization and gho controller for a quad rotor unmanned aerial vehicle. J. Elect. Eng., 57 (1): 20-27.

3. Hedrick, J.K. and A. Girard, 2005. http://www.me.berkeley.edu/ME237/notes.html.

Control of Nonlinear Dynamic Systems: Theory and Applications.

4. Kravaris, C., P. Daoutidis and R.A. Wright, 1994. Output feedback control of nonminimum phase nonlinear processes. Chem. Eng. Sci., 49 (13): 2107-2122.

5. Hassanzadeh, I. and S. Mobayen, 2007. Tuning of PID controllers for a robot using binary genetic algorithm. The 5th IFAC Intl. WS DECOM-TT 2007, Cesme, Turkey.

6. Hassanzadeh, I., S. Mobayen and H. Kharrati, 2007. Design of MIMO controller for a manipulator using tabu search algorithm. International conference on intelligence and Advanced Systems, 25-28. Kuala Lumpur, Malaysia.

7. Iraj Hassanzadeh and Saleh.Mobayen, 2007. Optimum design of pid controller for 5-bar-linkage manipulator using particle swarm optimization. Proceeding of the 4th International Symposium on Mechatronics and its Applications (ISMA07), Sharjah, UAE, 26-29.

8. Astrom, K.J. and K. Furuta, 2000. Swinging-up a Pendulum by Energy Control. Automatica, 36, pp: 278-285.

9. Yavin, Y., 1999. Control of a Rotary Inverted Pendulum. Elsevier. Applied Mathematics Letters, 12 (2): 131-134(4). 
10. Yan, Q., 2003. Output Tracking of Undergraduated Rotary Inverted Pendulum by Nonlinear Controller. Proceeding of the 42nd IEEE conference on Decision and Control, Maui, Hawaii USA.

11. Srinivasan, B., K. Guemghar, P. Huguenin and D. Bonvin, 2002. A Global Stabilization Strategy for an Inverted Pendulum. IFAC World Congress, Barcelona, Spain, pp: 1224.

12. Saleh Mobayen, 2007. A new binary coded ant algorithm for optimizing continuous functions. Int. J. Systemics, Cybernetics and Inform., 19-22 (ISSN 0973-4864).
13. Krohling, R.A. and J.P. Rey, 2001. Design of optimal disturbance rejection PID controllers using genetic algorithms. IEEE Trans. Evol. Comput., 5: 78-82.

14. Ziegler, J.G. and N.B. Nichols, 1942. Optimum Settings for Automatic Controllers, ASME Trans., 64, 759-768

15. Vakil-Baghmisheh, M.T. and Saleh Mobayen, 2007. A new general purpose binary coded ant algorithm. Proceeding of 15th Iranian Conference on Electrical Engineering, Tehran, Iran, pp: 117. 